\title{
O uso da tecnologia para a segurança do paciente
}

\author{
The use of technology for the safety of the patient
}

\section{EI uso de la tecnología en la seguridad del paciente}

Silvia Helena de Bortoli Cassiani', Fernanda Raphael Escobar Gimenes"', Aline Aparecida Silva Monzanil'I

\author{
' Professor Titular da Escola de Enfermagem de Ribeirão Preto da Universidade de São Paulo. Email: shbcassi@eerp.usp.br. \\ " Mestre em Enfermagem Fundamental e Docente da UNICASTELO. Email: fer_gimenes@yahoo.com.br. \\ III Mestre em Enfermagem Fundamental. Coordenadora de Auditoria e Central Ativa de Documentos do Hospital Vera Cruz. Email: \\ aline.monzani@uol.com.br.
}

\section{RESUMO}

O processo de utilização de medicamentos é complexo, multidisciplinar e carregado de aspectos psicológicos, tornando os erros de medicação frequentes e com sérias consequências para os clientes e organizações hospitalares. Neste contexto, a qualidade do cuidado e a segurança dos pacientes assumem papel de relevância na literatura nacional e internacional, sendo reconhecida como a Era da Segurança. Como estratégias para a redução e prevenção dos erros de medicação, o investimento em tecnologias tem sido amplamente discutido. Desta maneira, o texto tem como objetivo discutir a importância do uso da tecnologia para a seguranca dos pacientes nos hospitais brasileiros, no que concerne à utilização dos medicamentos. Dentre estas tecnologias, discutimos a utilização da prescrição médica eletrônica, dos códigos de barra, da dispensação e distribuição de medicamentos por dose unitária e das bombas de infusão "inteligentes". I nvestir em tecnologia para aprimorar os ambientes de trabalho e a segurança dos pacientes é uma questão que envolve negociação, considerando os custos advindos dos erros médicos e dos sistemas de medicação. Tais investimentos podem contribuir com o cuidado prestado ao paciente, diminuindo o tempo de internação, além de manter a força de trabalho qualificada e satisfeita.

Descritores: Sistemas de medicação; Tecnologia biomédica; Erros de medicação.

\section{ABSTRACT}

The process of medicament use is complex, multidisciplinary and full of psychological aspects, making kind of frequent the mistakes in medicaments with serious consequences to clients and hospital organizations. In that context, the care quality and the safety of the patients take over an important part in national and international literature, being recognized as the Safety Era. As reduction and prevention strategies of the mistakes in medicaments, the investment in technologies has been widely discussed. Thus, the text aims to discuss the importance of using technology for patient safety in Brazilian hospitals, concerning the use of medications. Among these technologies, we will discuss the use of computerized physician order entry, bar codes, dispensing and distribution of medicines by unit-dose and the "smart pumps". Investing in technology to improve the working environments and patient safety is an issue that involves negotiation, considering the costs arising from medical errors and medication systems. Such investments can help with the care provided to patients, reducing the time of admission, in addition to keeping the workforce skilled and satisfied.

Descriptors: Medication systems; Bbiomedical technology; Medication errors.

\section{RESUMEN}

El proceso de utilización de medicamentos es complejo, multidisciplinar y cargado de aspectos psicológicos, tornando los errores de medicación frecuentes y con serias consecuencias a los clientes y las organizaciones hospitalares. En este contexto, la calidad del cuidado y la seguridad de los pacientes asumen un papel importante en la literetura nacional e internacional, siendo reconocida como la Era de la Seguridad. Como estrategias a la reducción y prevención de los errores de medicación, la inversión en tecnología ha sido ampliamente discutida. Así, el texto tiene como objetivo discutir la importancia de utilizar la tecnología para la seguridad de los pacientes en hospitales de Brasil, sobre el uso de medicamentos. Entre estas tecnologías, analizar el uso de la prescripción médica electrónica, códigos de barras, la distribución y dispensación de la unidad de dosis de drogas y la bomba de infusión "inteligente". Invertir en tecnología para mejorar los entornos de trabajo y la seguridad de los pacientes es una cuestión que implica la negociación, teniendo en cuenta los costes derivados de los errores médicos y los sistemas de medicación. Estas inversiones pueden ayudar a la atención prestada a los pacientes, reducir el tiempo de admisión, además de mantener la mano de obra calificada y satisfecha.

Descriptores: Sistemas de medicación; Tecnología biomédica; Errores de medicación. 


\section{NTRODUÇÃO}

Várias são as questões de ordem estrutural presentes hoje na realidade de muitos países no que concerne ao setor saúde, tais como as mudanças demográficas decorrentes do envelhecimento e do declínio imediato ou futuro da população economicamente ativa, as dificuldades de equacionamento do financiamento e gastos públicos, o diferente desenvolvimento econômico das nações, a internacionalização da economia e da indústria, a avaliação do custo/eficácia e contenção de gastos no setor saúde bem como as alterações significativas nas tecnologias disponíveis nos cuidados médicos alterando o perfil de provisão de serviços com impactos na criação de novas demandas e necessidades de financiamento ${ }^{(1)}$.

A qualidade do cuidado e a segurança dos pacientes, nesse contexto, assumem papel de relevância, sendo reconhecida como a Era da Segurança. A Organização Mundial da Saúde, por meio da Resolução WHA 55.18, requer que os Estados Membros voltem a sua atenção à segurança dos pacientes, que desenvolvam normas e padrões globais, que promovam um quadro de políticas baseadas em evidências e mecanismos para reconhecer a excelência na segurança do paciente internacionalmente e que encorajem a pesquisa.

Ainda, em resolução, informa que o problema não é novo e que, embora os sistemas de saúde difiram de um país para outro, as ameaças à segurança do paciente têm causas e soluções freqüentemente similares. Informa, também que "A situação dos países em desenvolvimento e daqueles em fase de transição econômica merecem atenção particular por vários motivos: qualidade dos medicamentos, abastecimento, desempenho ruim de pessoal devido à falta de motivação, habilidades técnicas insuficientes e falta de financiamento dos custos operacionais essenciais dos serviços de saúde. Havendo, portanto, probabilidade dos eventos adversos serem muito maiores em detrimento das nações industrializadas".

De acordo com o relatório "To Err is human", publicado em 1999 pelos Institutos Americanos de Medicina, cerca de 44.000 a 98.000 pacientes morrem a cada ano naquele país devido às iatrogenias médicas e que 7.000 delas estão relacionadas aos erros de medicação(2).

A morbi-mortalidade relacionada ao uso de medicamentos tem custado mais de $\$ 136$ bilhões por ano aos Estados Unidos, sendo que $60 \%$ destes custos poderiam ter sido evitados ${ }^{(3)}$.

Os erros de medicação podem ocorrer em qualquer etapa da terapia medicamentosa, quais sejam: prescrição, transcrição, dispensação e distribuição, preparo e administração e monitorização.

Supõe-se que, nos países em desenvolvimento, mais especificamente no Brasil, a frequência dos erros de medicação seja ainda maior por diversos motivos, quais sejam: a falta de políticas sérias capazes de regulamentar a utilização dos medicamentos disponíveis atualmente no mercado, a incapacidade dos órgãos públicos em identificarem e gerenciarem informações acerca das reações adversas aos medicamentos e a falta de incentivo aos profissionais em notificarem os eventos adversos e os erros de medicação, problema este ligado a cultura de punição da grande maioria das instituições de saúde.

Mas, por que os erros de medicação ocorrem? A abordagem atual dos erros focaliza o problema no sistema e não simplesmente em responsabilizar os profissionais pelos erros. Ambientes de trabalho que prezam pela segurança dos pacientes são aqueles que educam seu pessoal, criam uma cultura em que a culpa não tem lugar, simplificam e padronizam processos e introduzem a verificação constante a fim de interceptar os possíveis erros antes que eles atinjam o paciente ${ }^{(4)}$.

Nós não podemos mudar a condição humana, mas nós podemos mudar as condições em que os humanos trabalham. Portanto, as pessoas precisam entender que os erros são sintomas de um sistema doente e que os esforços para a prevenção devem estar focados nas fraquezas deste sistema, e não nos indivíduos ${ }^{(5)}$.

Várias são as medidas já referenciadas na literatura como estratégias para a redução e prevenção dos erros de medicação nas instituições hospitalares, sendo uma delas o investimento em tecnologias tais como a implantação da prescrição médica eletrônica, do código de barras, da automatização da dispensação e do sistema de dispensação de medicamentos por dose unitária, além do uso de bombas de infusão "inteligentes"(6).

Sendo assim, este texto tem como objetivo discutir a importância do uso da tecnologia nos hospitais brasileiros e a segurança dos pacientes, no que concerne à utilização dos medicamentos.

\section{A PRESCRIÇÃo MÉdICA ELETRÔNICA NA SEGURANÇA DO PACIENTE}

As prescrições médicas eletrônicas são aquelas nas quais se utiliza um sistema computadorizado, de digitação, seguindo um modelo de disposição de dados. Elas podem variar em seu formato, desde sistemas bem estruturados e que alertam o prescritor quanto às alergias, interações medicamentosas e doses máximas, até sistemas mais simples onde as informações são digitadas pelo médico ou transcritor e enviadas diretamente, ou por fax, à Farmácia ${ }^{(7)}$.

Elas ampliam a segurança dos medicamentos porque são mais estruturadas, legíveis e muitas informações podem ser fornecidas ao prescritor durante elaboração da prescrição. Além de 
possibilitarem que o erro seja corrigido no momento da digitação sem que, para isso, haja rasuras ou rabiscos, os quais dificultam ainda mais o seu entendimento ${ }^{(8)}$.

Apesar de algumas pesquisas demonstrarem as suas vantagens, como a redução dos erros em mais da metade, a maioria dos hospitais no mundo nao adota esta tecnologia devido aos altos custos referentes à implementação e manutenção do sistema. Dados de um estudo realizado em hospital americano revelaram valores na ordem de US\$1,9 milhões para implementá-lo e cerca de US\$ $500.000 /$ ano para mantê-lo ${ }^{(9)}$.

Estudo brasileiro, realizado em 2005, analisou 1.425 prescrições médicas em cinco hospitais, todos membros da Rede Sentinela da Anvisa. No entanto, apenas um hospital apresentava o sistema computadorizado de prescrições e os medicamentos eram todos prescritos pelo nome genérico. Embora o sistema de prescrição nesse hospital seja computadorizado, ele permite que o médico prescreva medicamentos manualmente, principalmente nos casos onde o estado geral do paciente é alterado, necessitando acrescentar novos fármacos ou suspender outros ${ }^{(10)}$.

Apesar do sistema computadorizado de prescrições representar um grande avanço dentro das estratégias utilizadas para minimizar erros decorrentes de prescrições mal formuladas, ele não erradica a possibilidade de ocorrência de erros de medicação, visto que outros podem surgir.

\section{UTI LIZAÇÃO DE CÓDIGOS DE LEITURA POR MÁQUI NAS NA I DENTI FI CAÇÃO DO PACI ENTE}

A administração de medicamentos a pacientes errados é um dos erros de medicação que frequentemente ocorre nos hospitais, tendo como uma de suas causas falhas na identificação dos pacientes. Este problema poderia ser minimizado simplesmente com 0 uso de pulseiras de identificação. No entanto, estudos revelaram que, apesar dos pacientes apresentarem tais pulseiras, os erros ainda ocorriam ${ }^{(11)}$.

Uma maneira eficiente de resolver este problema seria a utilização de códigos de leitura óptica por máquinas, ou seja, a implantação do código de barra.

A tecnologia de códigos de barra tem sido amplamente utilizada nas indústrias, e alguns hospitais americanos já a tem implantado com sucesso, estando ela relacionada a uma redução de $80 \%$ nos erros de administração de medicamentos. Além de permitir que se tenha certeza de que o medicamento em mãos é, de fato, o prescrito podendo também registrar quem administrou e quem recebeu em vários intervalos de tempo(8).

Os códigos de barra são mais rápidos do que os olhos humanos e também mais precisos, permitindo a ocorrência de menos de um erro para cada mil leituras. Além disso, não se cansam ou se distraem ${ }^{(12)}$.

A Comissão de Acreditação Hospitalar Americana requer como meta número um das instituições a melhoria da exatidão da identificação de pacientes. Em fevereiro de 2004, o Food and Drug Aministration estipulou o prazo final para que as indústrias acrescentem código de barras nos medicamentos.

A desvantagem desta tecnologia é o seu alto custo, estimado na ordem de U\$200.000 dólares a U\$ 1 milhão, dependendo do tamanho do hospital. Ressalta-se, ainda, a importância de manter o sistema atualizado para que a transmissão dos dados das pulseiras dos pacientes e dos medicamentos ocorra eficientemente para o sistema computacional.

\section{A AUTOMATIZAÇÃO DA DISPENSAÇÃO E O SISTEMA DE DISTRIBUIÇÃO DE MEDI CAMENTOS POR DOSE UNITÁRI A}

A implantação de um sistema de dispensação de medicamentos seguro, organizado e eficiente é essencial para a redução de custos e para assegurar que a prescrição médica seja seguramente cumprida. Desta maneira, um sistema de dispensação apropriado é um aliado importante na prevenção e na redução dos erros de medicação, uma vez que diminuem as oportunidades de erros durante a dispensação das doses na farmácia ${ }^{(13)}$.

Além disso, o envolvimento atento do farmacêutico na dispensação, bem como sua função educativa, é de valia no suprimento de informações ao médico assistente ou ao corpo clínico da instituição, representando uma das últimas oportunidades de identificar, corrigir ou reduzir possíveis riscos associados à terapêutica. Suas responsabilidades, no momento da dispensação, são múltiplas, envolvendo questões de cunho legal, técnico e clínico ${ }^{(14)}$. No entanto, no Brasil, o farmacêutico hospitalar não está presente em 25\% das farmácias hospitalares brasileiras ${ }^{(15)}$.

Estudo realizado em quatro hospitais brasileiros identificou que, pelo menos, $90 \%$ dos medicamentos eram distribuídos por meio do sistema individualizado, e que nenhum deles apresentava o sistema de dose unitária ${ }^{(16)}$.

A distribuição por dose individualizada diminui os prejuízos financeiros à instituição causados por vencimento das datas de validade, acondicionamento inadequado, pelo distanciamento entre o gasto com a aquisição e recebimento pelo uso do medicamento, sem falar na menor probabilidade de ocorrer erros na administração devido à grande disponibilidade e variedade de medicamentos ${ }^{(1)}$. No Brasil, $34,8 \%$ dos hospitais fazem uso deste sistema ${ }^{(17)}$.

No sistema de dose unitária, o fármaco é dispensado na dose exata de acordo com a prescrição médica, não requerendo manipulação e/ou reconstituição do medicamento pela equipe de 
enfermagem e nem realização de cálculos matemáticos. Este sistema permite que a equipe de enfermagem administre a dose correta, minimizando os riscos de eventos adversos, além de minimizar o tempo gasto pelos profissionais com o preparo das drogas.

\section{O USO DE SISTEMAS “I NTELI GENTES" DE BOMBAS DE I NFUSÃO}

Apesar das diversas tecnologias implementadas nas instituições hospitalares terem demonstrado redução significativa dos erros de medicação, elas têm apresentado pouco ou nenhum impacto nos erros associados à administração intravenosa de medicamentos. Estas iatrogenias podem resultar em graves eventos adversos aos pacientes ${ }^{(6)}$. Os problemas com dispositivos de infusão, sozinhos, são responsáveis por, aproximadamente, 35\% de todos os erros de medicação(18).

Uma solução plausível, de acordo com a literatura, seria a utilização de um sistema de bombas de infusão "inteligentes", a qual tem se mostrado capaz de detectar muitos dos erros envolvendo a administração de medicamentos por via intravenosa, além de ter o potencial de reduzir as freqüências de erros graves.

As bombas de infusão "inteligentes", geralmente têm a habilidade de fornecer as seguintes vantagens: (a) apresenta uma espécie de biblioteca "on-board" com informações acerca do medicamento; (b) auxílio do fabricante com a execução do sistema; (c) contínua exposição do fármaco, da dose prescrita, e de todos os cuidados com a administração do medicamento, com limites para doses insuficientes ou sobre-doses; e (d) uma série de alertas de registros da administração dos medicamentos e ações corretivas que devem ser tomadas ${ }^{(19)}$.

Os fabricantes destas bombas "inteligentes" afirmam que elas podem reduzir um erro potencialmente fatal a cada 2.6 dias em um hospital de 350 leitos $^{(20)}$.

Nos Estados Unidos, $41 \%$ dos hospitais já fazem uso desta tecnologia(20), todavia, no Brasil, estas bombas ainda não estão presentes na grande maioria das instituições hospitalares.

\section{CONCLUSÃO}

Sabe-se que incorporar avanços tecnológicos no cuidado direto ao paciente não é tarefa fácil para a equipe de saúde. Entretanto, todos reconhecem que são ferramentas fundamentais a serem utilizadas na redução dos erros e, consequentemente, na prevenção de eventos adversos, melhorando a qualidade e a segurança do cuidado prestado ao paciente hospitalizado.

Estas inovações tecnológicas são possíveis, executáveis e com custo-benefício efetivo. As organizações de saúde devem fazer uma análise sistemática do processo de medicação, identificando suas vulnerabilidades, com o intuito de implantar medidas efetivas para sanar seus problemas. A implantação efetiva destes processos, além da atenção especial em cada etapa do sistema de medicação pode levar a mudanças significativas e com resultados positivos na redução dos erros, garantindo mais segurança aos pacientes.

Investir em tecnologia para aprimorar os ambientes de trabalho e a segurança de pacientes é uma questão que envolve negociação, considerando os custos advindos dos erros médicos e dos sistemas de medicação. Tais investimentos podem contribuir com o cuidado prestado ao paciente, diminuindo o tempo de internação, além de manter a força de trabalho qualificada e satisfeita.

\section{REFERÊNCIAS}

1. Silva AEBC. Análise do sistema de medicação de um hospital universitário do Estado de Goiás [dissertation]. Ribeirão Preto: Escola de Enfermagem/USP; 2003. 155p.

2. Leape LL, Berwick DM, Bates DW. What practices will most improve safety? Evidence-based medicine meets patient safety. JAMA. 2002;288(4):501-7.

3. Kohn LT, Corrigan JM, Donaldson MS. To err is human: building a safer health system. 3rd ed. Washington: National Academy of Institute of Sciences; 2000.

4. Chaiken BP, Holmquest DL. Patient safety: modifying processes to eliminate medical errors. Nurs Outlook. 2003;51(3): S21-4.

5. Reason J. Human error: model and management. BMJ . 2000; 320(7237): 768-70.

6. Rothschild JM, Keohane CA, Cook EF, Orav EJ, Burdick $E$, Thompson $S$, et al. A controlled trial of smart pumps to improve medication safety in critically ill patients. Crit Care Med. 2005;33(3):53340.

7. Freire CC, Gimenes FRE, Cassiani SHB. Análise da prescrição médica informatizada em duas clínicas de um hospital universitário. Medicina (Ribeirão Preto). 2004;37(1/2): 91-6.

8. Bates DW. Using information technology to reduce rates of medication errors in hospitals. BMJ. 2000; 320(7237) : 788-91.

9. Glaser J, Teich JM, Kuperman G. Impact of informations events on medical care. In: Proceedings of the 1996 HIMMS Annual Conference. Chicago: Healthcare Information and Management Systems Society; 1996. p.1-9.

10. Gimenes FRE. A segurança de pacientes na terapêutica medicamentosa: análise da redação da prescrição médica nos erros de administração de medicamentos em unidades de clínica médica [dissertation]. Ribeirão Preto: Escola de Enfermagem/USP; 2007. 114p.

11. Miasso Al, Cassiani SHB. Erros na administracao 
de medicamentos: divulgacao de conhecimentos e identificacao do paciente como aspecto relevante. Rev. esc. enferm. USP. 2000; 34(1): 16-25.

12. Davis NM. Initiatives for reducing medication errors: the time is now. Am J Health Syst Pharm. 2000; 57(16): 1487-92.

13. Anacleto TA, Perini E, Rosa MB. Medication errors and drug-dispensing systems in a hospital pharmacy. Clinics. 2005; 60(4):325-32.

14. Pepe VLE, Osório de Castro CGS. A interação entre prescritores, dispensadores e pacientes: informação compartilhada como possível benefício terapêutico. Cad. Saúde Pública. 2000; 16(3): 815-22. 15. Osório de Castro CGS, Castilho SR. Diagnóstico da Farmácia Hospitalar no Brasil. Rio de Janeiro: Fundação Oswaldo Cruz; 2004.

16. Cassiani SHB, Miasso Al, Silva AEBC, Fakin FT, Oliveira RC. Aspectos gerais e número de etapas do sistema de medicação de quatro hospitais brasileiros. Rev Latino-am Enfermagem. 2004; 12(5): 781-9.

17. Anacleto TA, Perini E, Rosa MB, César CC. Medication errors and drug-dispensing systems in a hospital pharmacy. Clinics. 2005;60(4):325-32.

18. Riddell B. "Smart" infusion pumps and the clinical nurse specialist. Clin Nurse Spec. 2006;20(3):11718.

19. Rosenfeld K. Smart pumps help crack the safety code. Nurs Manage. 2004; 35(5): 49-52.

20. Ritter T. Perspectives from ECRI: Infusion pump error reduction. J Clin Eng. 2005; 30(2):81-2.

Artigo recebido em 15.07.08.

Aprovado para publicação em 25.05.09. 